\title{
The Simulation Study of the Spread-Spectrum System
}

\author{
MingE Sha ${ }^{1, a}$, Yonggang $\mathrm{Xie}^{2, \mathrm{~b}^{*}}$ \\ ${ }^{1}$ Department of Mathematics, Kunming University, Kunming, 650214, CHINA \\ ${ }^{2}$ Equipment Management Department of Kunming University, Kunming, 650214, CHINA

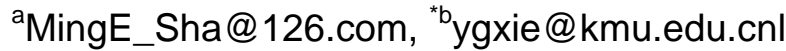

Keywords: Simulation, Spread-Spectrum, Direct-Sequence (DS), frequency hopping, FSK

\begin{abstract}
Spread-spectrum technology produces a transmitted spectrum much wider than the minimum bandwidth required and links as a means of overcoming intentional jamming. In this paper, we provide a brief outline of the theory of the spread-spectrum technique. Then we apply this technique to FSK system and give simulation results.
\end{abstract}

\section{Introduction}

The spread-spectrum Technique (SST) was first published by Hollywood actress Hedy Lamarr and pianist George Antheil in 1941[1]. They identified jamming of Allied radio communications by the Axis as a particular problem, and developed spread-spectrum and frequency hopping technology to defeat it [2].

SST is an important encoding method for wireless communications, which gain more and more attentions in recent decades. This technology spreads data over wide bandwidth and makes jamming and interception harder.There are different ways to generate spread-spectrum signals, for example, direct sequence (DS), frequency hop (FH), time hop (TH), and multicarrier (MC). The first major application of SST arose during the mid-sixties, when NASA employed the method to precisely measure the range to deep space probes. Recent years, typical applications of SST including: satellite-positioning systems (GPS), multiple access, 4G, IEEE 802.11 secure communications and so on.

In this paper, we give literature study of the spread-spectrum technology and simulate the direct-sequence spread-spectrum system. The remainder of this paper is organized as follows: the principle of the SST is described in Section II. Section III presents different types of SST. The analysis of system performance and simulation results is given in Section VI. Section V concludes the paper.

\section{The Principle of the SST}

Spread-spectrum (SS) is a technique which transmitting signals occupy a much greater band than the minimum necessary band to send the original signals. At the receiver, the received SS signals is despreading. The utility of spread spectrum technique can be illustrated easily using the famous Shannon and Hartley channel-capacity theorem [3], since this is most directly related to the problem of signal-to-noise ratio.

$$
C=B \times \log _{2}\left(1+\frac{S}{N}\right)
$$

where, $C$ is the channel capacity in bits per second (bps). It is the maximum data rate for a theoretical BER; $B$ is the bandwidth of the channel in Hz. It is the price to be paid, because frequency is a limited resource. $S$ is the average received signal power over the bandwidth measured in Watts. $N$ is the average noise or interference power over the bandwidth, measured in watts. And $S / N$ is the signal-to-noise power ratio (SNR) or the carrier-to-noise power ratio (CNR) of the communication signal to the Gaussian noise interference, which describes the environmental conditions or the physical characteristics. This approach says that one can maintain or even increase communication 
performance (high $C$ ) by allowing or injecting more bandwidth (high $B$ ), even when signal power is below the noise floor, which means we can trade signal to noise ratio for bandwidth, or vice versa.

\section{Types of Spread SST}

Different SST are distinguished according to the point in the system at which a Pseudo-Random Number (PRN) is inserted in the communication channel [4].

- If the PRN is inserted at the data level, this is the direct-sequence form of spread-spectrum (DSSS).

- If the PRN acts at the carrier-frequency level, this is the frequency-hopping form of spread-spectrum (FHSS).

- If the PRN acts as an on/off gate to the transmitted signal, this is a time-hopping spread-spectrum technique (THSS). There is also the "chirp" technique, which linearly sweeps the carrier frequency in time.

We can mix two or all the above techniques to form a high-performance hybrid spread-spectrum technique, such as DSSS \& FHSS, DSSS \& FHSS, and DSSS \& FHSS \& THSS. In this paper, we use DSSS.

\section{The Simulation Results of the FSK SS System}

In this section, we simulation study of the FSK SS system, in which, only single frequency point are simulated. The steps of the simulation are as following:

- $\quad$ Initialization $(\mathrm{Ts}=0.00001$; EndTime = 2-Ts);

- $\quad$ Generate NRZ information code;

- $\quad$ FSK modulation and Low-pass filtering;

- Spreading and Band-pass filtering;

- $\quad$ Adding Gaussian white noise;

- $\quad$ Despreading and FSK demodulation;

- Low-pass filtering and getting lower frequency

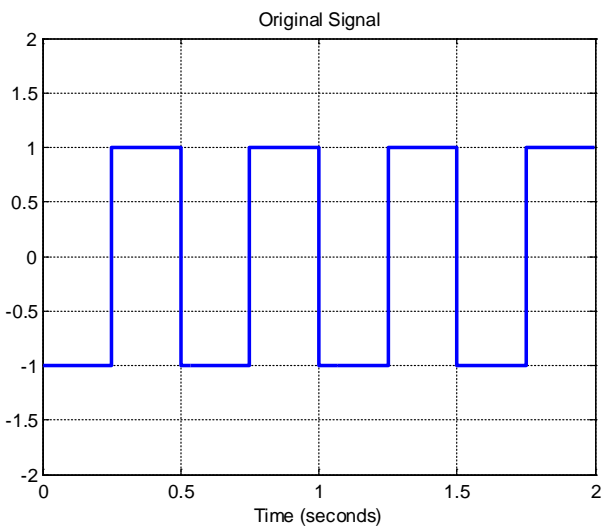

Fig. 1 Generation of the original signal

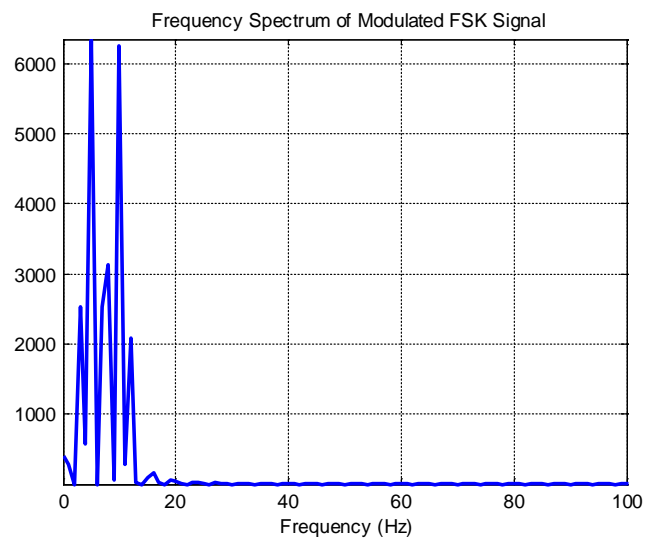

Fig. 2 Frequency spectrum of modulated FSK signal

At first, we generate the binary RNZ code (Fig. 1) and using FSK modulated the original signal (Fig. 2). Then let the modulated FSK signal pass through the LPF to eliminate the outside the band noise. The time domain FSK wave which has gone through the LPF is show in Fig. 3. Fig. 4 shows the frequency spectrum of the LPF FSK wave. 


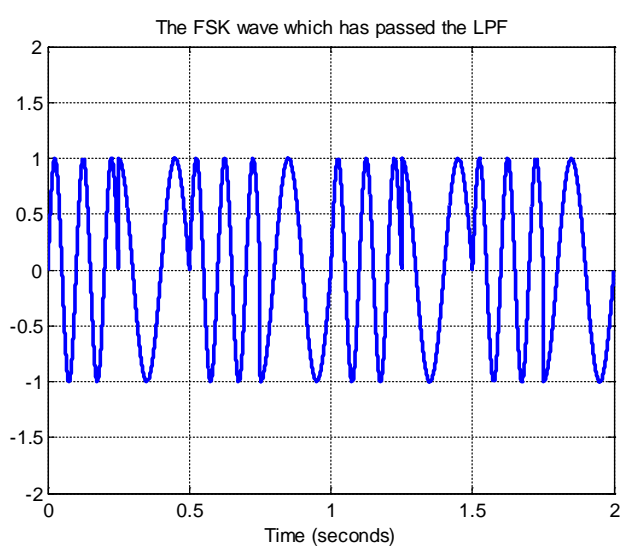

Fig. 3 The LPF FSK wave

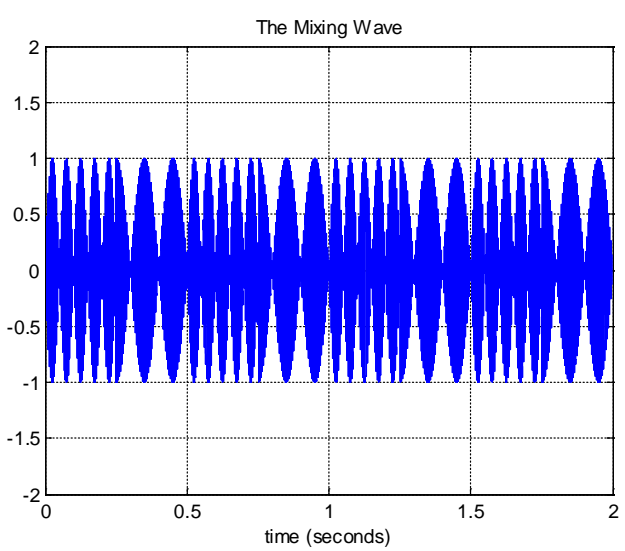

Fig. 5 The Mixing Wave

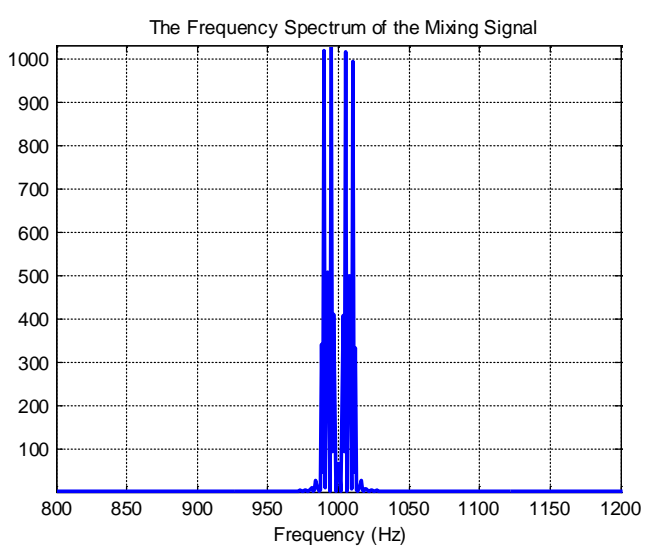

Fig. 7 The Frequency spectrum of the mixing signal

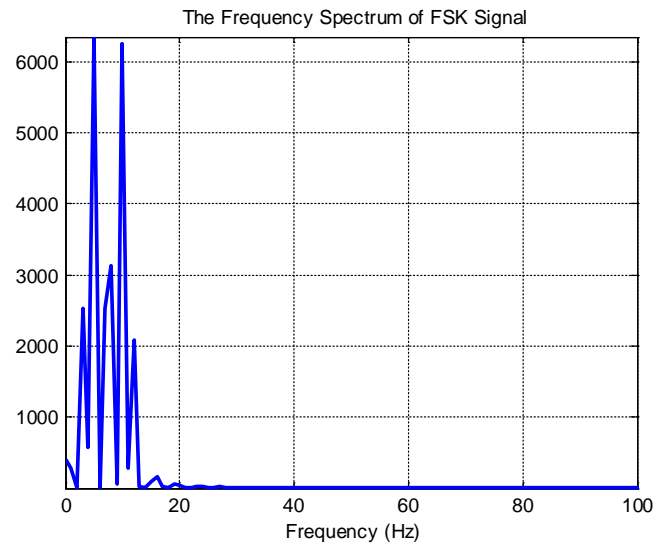

Fig. 4 The frequency spectrum of LPF FSK signal

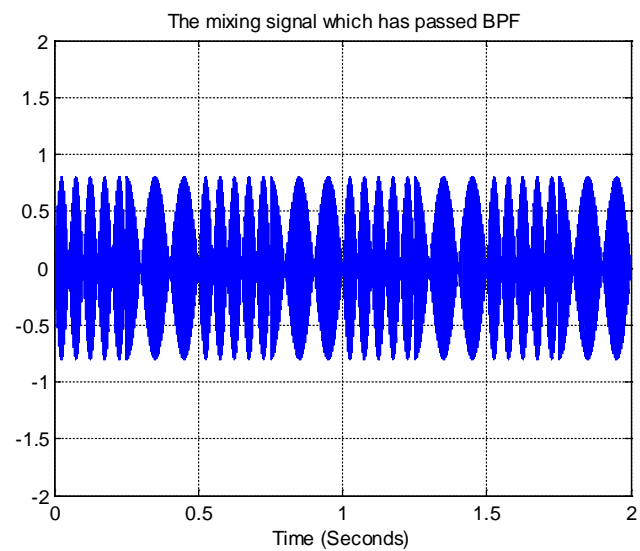

Fig. 6 The mixing signal which has passed bandpass filter

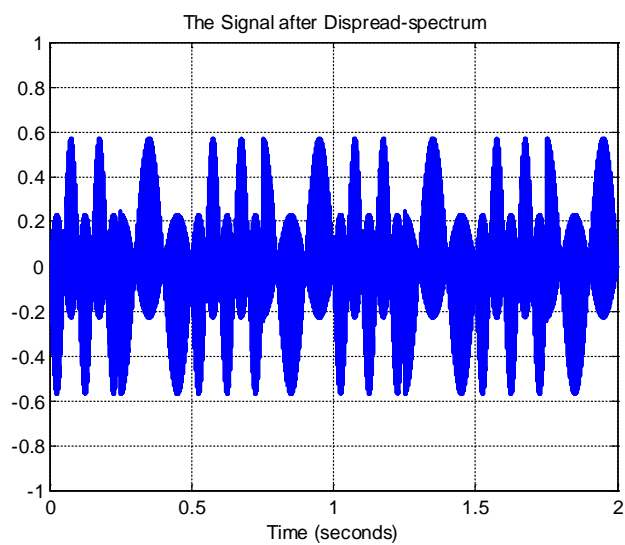

Fig. 8 The signal after dispread-spectrum

Fig. 5 shows the mixing wave, which is the spread-spectrum process, after spreading, the mixing signal is through BPF (Fig. 6) to improve the signal's quality. The frequency spectrum of the signal, which after the bandpass filter is shown in Fig. 7. Fig. 8 is the spectrum of the dispread-spectrum, and the lower frequency of the dispread-spectrum signal is show in Fig. 9. Fig.10 is the signal before sampling decision. The Fig. 10 is the recovered signal. Compared with the Fig. 1, we can see the recovered signal has the same wave as the original signal, which proved the effective of the SS system. 

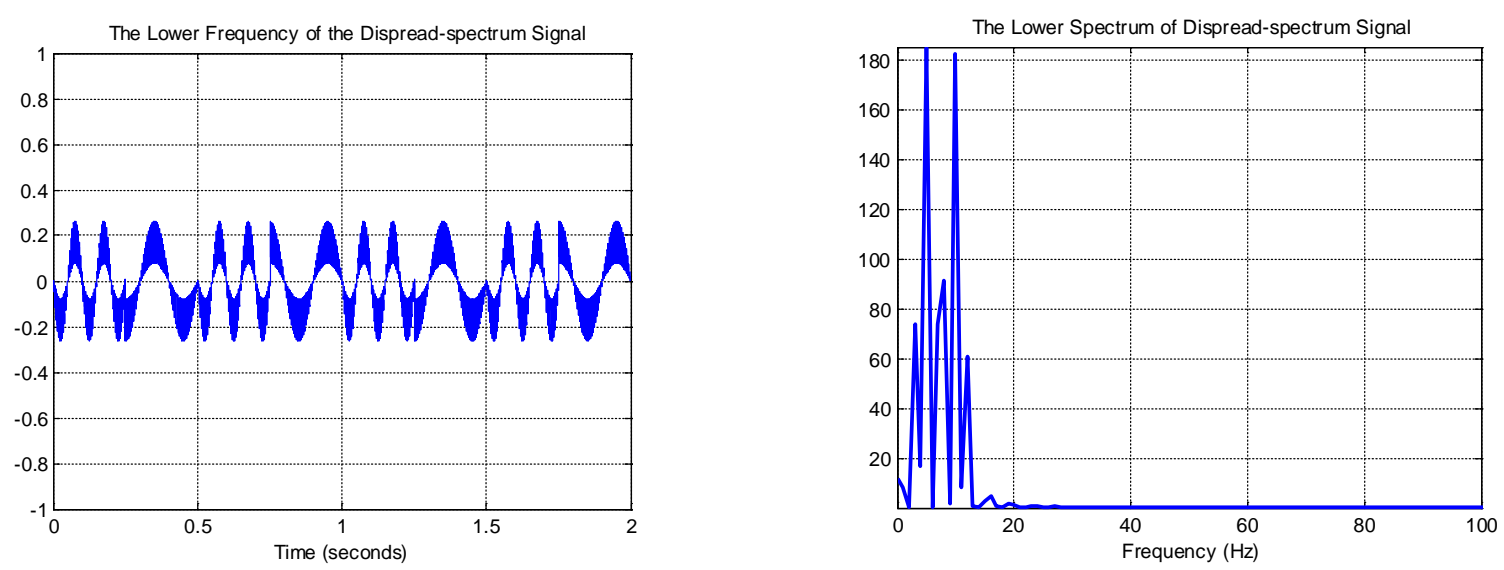

Fig. 9 The lower frequency of the dispread-spectrum signal

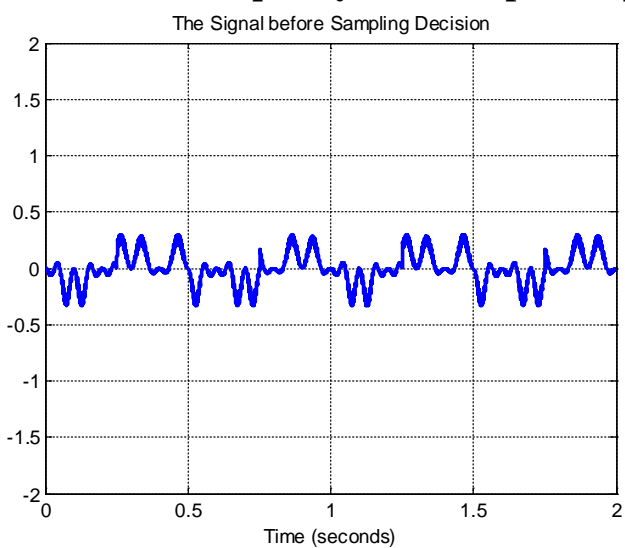

Fig. 9 The signal before sampling decision
Fig. 8 The signal after dispread-spectrum

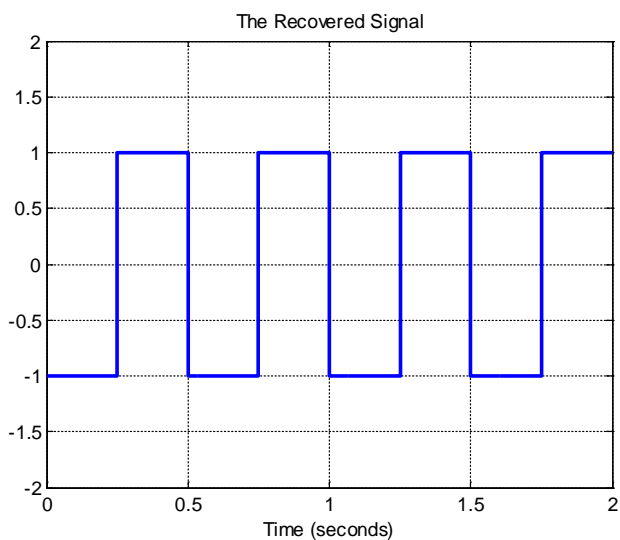

Fig. 10 The recovered signal

\section{Conclusion}

SST are the technology which can overcome a jamming situation, and very useful when an adversary intends to disrupt the communication. A good SS system should be difficult to detect by an adversary, and the signal need be difficult to disturb with a jamming signal. In this paper, we use DSSS to spread FSK signal and transmit the modulated signal in the AWGN channel, at the receiver, demodulate and despread the received signal and compare it with the original signal. From the results, we can see the SST is an effective and reliable technology to transmit signals.

\section{References}

[1] "Movie Legend Hedy Lamarr to be Given Special Award at EFF's Sixth Annual Pioneer Awards” (Press release). Electronic Frontier Foundation. 11 March 1997. Retrieved 1 February 2014. [2] Robert A. Scholtz. "Notes on spread-spectrum history". IEEE Transactions on Communications, vol. 31, no. 1, pp. 82 - 84, January 1983.

[3] Robert A. Scholtz. "The origins of spread-spectrum communications”. IEEE Transactions on Communications, vol. 30, no. 5, pp. 822 - 854, May 1982.

[4] “An Introduction to Direct-Sequence Spread-Spectrum Communications”. Published on Oct 17, 2011 Telecommunication Systems Published in: Technology, Business.

[5] M. B. Pursley, "Performance evaluation for phase-coded spread-spectrum multiple-access communication- Part I: System analysis,” IEEE Trans. Commun., vol. 25, no. 8, pp. 795-799, Aug. 1977.

[6] R. A. Scholtz, "Multiple access with time-hopping impulse modulation,” Proc. MILCOM '93, pp. 11-14, Boston, MA, Oct. 1993. 